\title{
On expansion parallax distances for planetary nebulae
}

\author{
G. Mellema $a^{\star \star \star}$ \\ Sterrewacht Leiden, PO Box 9513, 2300 RA, Leiden, The Netherlands \\ Received 10 October 2003 / Accepted 2 December 2003

\begin{abstract}
The distances to individual wind-driven bubbles such as Planetary Nebulae (PNe) can be determined using expansion parallaxes: the angular expansion velocity in the sky is compared to the radial velocity of gas measured spectroscopically. Since the one is a pattern velocity, and the other a matter velocity, these are not necessarily the same. Using the jump conditions for both shocks and ionization fronts, I show that for typical PNe the pattern velocity is 20 to $30 \%$ larger than the material velocity, and the derived distances are therefore typically 20 to $30 \%$ too low. I present some corrected distances and suggest approaches to be used when deriving distances using expansion parallaxes.
\end{abstract}

Key words. shock waves - planetary nebulae - stars: distances - stars: AGB and post-AGB - hydrodynamics ISM: planetary nebulae: general

\section{Introduction}

Distances to individual nebular objects, such as Planetary Nebulae (PNe) are important to quantitatively understand their structure and evolution, but notoriously difficult to determine. Typically uncertainties of a factor of two can be expected from so-called statistical methods, a review of which can be found in Terzian (1993).

Probably the best method for measuring the distance to an individual nebula is the "expansion parallax" method. Here the nebular expansion in the sky as measured from images taken at different epochs is compared to the radial velocity as measured spectroscopically. This method became feasible with the advent of high resolution imaging, in the late 1980's at radio wavelengths using interferometers (Masson 1986, 1989a,b; Hajian et al. 1993, 1995; Kawamura \& Masson 1996; Hajian \& Terzian 1996; Christianto \& Seaquist 1998) and nowadays also in the optical with the Hubble Space Telescope (HST, Reed et al. 1999; Palen et al. 2002; Li et al. 2002).

Clearly the simplest application of this method suffers from a number of drawbacks. One complication is the choice for the spectroscopic velocity, where different ions often give different values. The other is the assumption of spherical expansion. However, authors have been making corrections for the shape and aspherical expansion of the measured nebulae, and applying such sophisticated templates clearly has made the method more useful, in some cases reaching claimed errors as low as $10-20 \%$.

Still, the application requires a reasonably close or particularly rapidly expanding nebula and to date has only been applied to a limited number of PNe. Table 1 list all cases with

^ Present address: Netherlands Foundation for Research in Astronomy, PO Box 2, NL-7990 AA Dwingeloo, The Netherlands.

$\star \star$ e-mail: mellema@strw.LeidenUniv.nl a well determined expansion parallax distance, leaving out the cases where only upper limits were found.

Among the corrections needed to accurately use the expansion parallax method there is one which to date has received little attention in the literature, even though it is quite basic. This is the fact that the expansion velocity as measured in the sky is a pattern velocity, whereas the spectroscopically measured velocity is a material velocity, and the two are generally not the same. This effect was touched upon by Marten et al. (1993) and Steffen et al. (1997), but has not been taken into account in any of the published expansion parallax distances.

The extreme case would be that of an R-type ionization front making its way through a stationary medium, and clearly here the expansion parallax method becomes useless, as has been mentioned by various authors. Luckily, this situation is thought to be rare in PNe. However, also in the more common cases of shock fronts or slower moving D-type ionization fronts, the two velocities will differ. Although typically not by much, the effect is systematic, not random, and it should therefore be taken into account when using the expansion parallax method. Especially as the measurements are becoming more accurate over time, this factor can no longer be neglected.

In this paper I calculate the magnitude of the discrepancy between the two velocities for shocks (Sect. 2), ionization front structures (Sect. 3) and so-called "shells" (Sect. 4). In Sect. 5 I apply the derived corrections to the published results of the expansion parallax method and suggest strategies to optimize the expansion parallax method.

\section{Shock waves}

Since PNe are basically wind-driven bubbles, they contain shocks. Numerical hydrodynamic modelling has shown that in round or elliptical nebulae the inner bright rim is normally 
Table 1. Expansion parallaxes.

\begin{tabular}{lllll}
\hline \hline PN & $u_{\text {spectro }}\left(\mathrm{km} \mathrm{s}^{-1}\right)^{*}$ & Distance (kpc) & Radio/Optical & Reference \\
\hline BD+30 3639 & $22 \pm 4$ & $2.8_{-1.2}^{+4.7}$ & $\mathrm{r}$ & Masson (1989b) \\
BD+30-3639 & $22.0 \pm 1.5$ & $2.68 \pm 0.81$ & $\mathrm{r}$ & Hajian et al. (1993) \\
BD+30 3639 & $22 \pm 4$ & $1.5 \pm 0.4$ & $\mathrm{r}$ & Kawamura \& Masson (1996) \\
BD+30 3639 & 25.6 & $1.2 \pm 0.12$ & $\mathrm{o}$ & Li et al. (2002) \\
IC 2448 & $17.9 \pm 0.3$ & $1.38 \pm 0.4$ & $\mathrm{o}$ & Palen et al. (2002) \\
NGC 3242 & $26 \pm 4$ & $0.42 \pm 0.16$ & $\mathrm{r}$ & Hajian et al. (1995) \\
NGC 6210 & $23 \pm 5$ & $1.57 \pm 0.40$ & $\mathrm{r}$ & Hajian et al. (1995) \\
NGC 6543 & $16.4 \pm 0.16$ & $1.00 \pm 0.27$ & $\mathrm{o}$ & Reed et al. (1999) \\
NGC 6572 & $14 \pm 4$ & $1.49 \pm 0.62$ & $\mathrm{r}$ & Hajian et al. (1995) \\
NGC 6572 & $14 \pm 4$ & $1.2 \pm 0.4$ & $\mathrm{r}$ & Kawamura \& Masson (1996) \\
NGC 6578 & $19.2 \pm 0.5$ & $2.00 \pm 0.5$ & $\mathrm{o}$ & Palen et al. (2002) \\
NGC 6884 & $16.6 \pm 0.4$ & $2.20 \pm 0.8$ & $\mathrm{o}$ & Palen et al. (2002) \\
NGC 7027 & 21 & $0.94 \pm 0.2$ & $\mathrm{r}$ & Masson (1986) \\
NGC 7027 & $17.5 \pm 1.5$ & $0.88 \pm 0.15$ & $\mathrm{r}$ & Masson (1989a) \\
NGC 7027 & $17.5 \pm 1.5$ & $0.703 \pm 0.095$ & $\mathrm{r}$ & Hajian et al. (1993) \\
NGC 7662 & $21 \pm 7$ & $0.79 \pm 0.75$ & $\mathrm{r}$ & Hajian \& Terzian (1996) \\
VY 2-2 & $19.5 \pm 0.4$ & $3.6 \pm 0.4$ & $\mathrm{r}$ & Christianto \& Seaquist (1998) \\
\hline
\end{tabular}

* This is the spectroscopic velocity used by the authors, which can correspond to a shock or ionization front velocity.

associated with the shock wave being driven into the surrounding material by the stellar wind. Key papers describing the radiation-hydrodynamic evolution of PNe are Marten \& Schoenberner (1991); Mellema (1994, 1995), and a review is presented in Schönberner \& Steffen (2003). The application to an individual PN is shown in Corradi et al. (2000). Whenever I mention numerical simulations in what follows, I refer to these papers.

The jumps of density and velocity across a shock are given by the Rankine-Hugoniot conditions. These are usually given for the reference frame of the shock, in which case the velocity jump is

$\frac{v_{0}}{v_{1}}=\frac{(\gamma+1) \mathcal{M}^{2}}{(\gamma-1) \mathcal{M}^{2}+2}$

which for infinite Mach number $\mathcal{M}$ and an adiabatic index $\gamma=$ $5 / 3$ gives the classical value of 4 . The Mach number of the shock is given by

$\mathcal{M}=\frac{\left|v_{0}\right|}{a_{0}}$

where $a_{0}$ is the sound speed in the pre-shock gas, given by

$a_{0}=\sqrt{\frac{\gamma k T_{0}}{\mu m_{\mathrm{H}}}}$,

with $k$ the Boltzmann constant, $T_{0}$ the gas temperature, $\mu$ the mean molecular weight, and $m_{\mathrm{H}}$ the mass of atomic hydrogen.

However, in the stellar frame, the shock has a velocity which we will call $u_{\mathrm{s}}$ and the pre- and post-shock velocities are given by $u_{0,1}=v_{0,1}+u_{\mathrm{s}}$, see Fig. 1 .

The expansion parallax method measures $u_{\mathrm{s}}$ from the angular expansion of the shock front, $\dot{\theta}=u_{\mathrm{s}} / D$, and $u_{1}$ from the spectroscopy, and derives the distance $D$ from the ratio of the

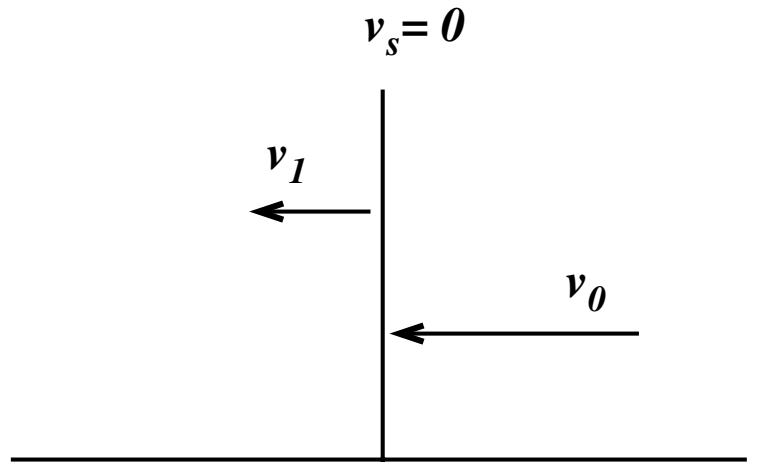

Shock Frame

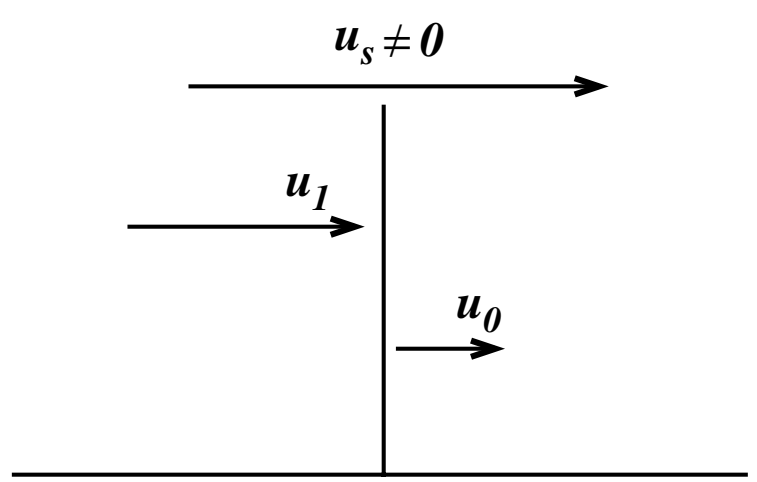

Stellar Frame

Fig. 1. Sketch of the shock configuration and the definition of the various velocities.

two, assuming $u_{\mathrm{s}}=u_{1}$. From the expression above it is immediately clear that $u_{\mathrm{s}}$ and $u_{1}$ are not equal. If the PN woud be expanding into complete vacuum the two velocities would be 
identical, but in the case where it is moving into a surrounding medium, it is more proper to view the expansion as a disturbance or wave travelling through the medium, raising its density and velocity. The speed at which the wave travels is not the same as the velocity to which the material is accelerated by the passage of the wave.

Let us consider the ratio $\mathcal{R}$ of the two velocities, $u_{\mathrm{s}} / u_{1}$. The distance to the PN can then be expressed as $\mathcal{R} u_{1} / \dot{\theta}$. Using the relations between $u_{0,1}$ and $v_{0,1}$ together with Eqs. (1) and (2) (realizing that in our shock frame both $v_{0}$ and $v_{1}$ are negative) gives

$\mathcal{R}=\frac{(\gamma+1) \mathcal{M} u_{0}+(\gamma+1) \mathcal{M}^{2} a_{0}}{(\gamma+1) \mathcal{M} u_{0}+2\left(\mathcal{M}^{2}-1\right) a_{0}}$.

The limits of this ratio are $\left(u_{0}+a_{0}\right) / u_{0}$ for $\mathcal{M} \rightarrow 1$ and $(\gamma+1) / 2$ for $\mathcal{M} \rightarrow \infty$. This shows that only for isothermal $(\gamma=1)$ hypersonic shocks the ratio tends to one for high Mach numbers. Choosing values for the pre-shock velocity $u_{0}$, pre-shock sound speed $a_{0}$, and shock Mach number $\mathcal{M}$ gives a value for $\mathcal{R}$. The material velocity $u_{1}$ can then be found from

$u_{1}=u_{0}+2 a_{0}\left(\frac{\mathcal{M}^{2}-1}{(\gamma+1) \mathcal{M}}\right)$.

Reversely, given values for $u_{0}, u_{1}$, and $a_{0}$, the shock's Mach number can be found from

$\mathcal{M}=\frac{(\gamma+1)\left(u_{1}-u_{0}\right)+\sqrt{(\gamma+1)^{2}\left(u_{0}-u_{1}\right)^{2}+16 a_{0}^{2}}}{4 a_{0}}$,

which can then be used to derive $\mathcal{R}$ using Eq. (4).

In Fig. 2 I plot the ratio $\mathcal{R}$ as function of $u_{1}$, for six preshock velocities $u_{0}$ in the range 1 to $25 \mathrm{~km} \mathrm{~s}^{-1}$, using a $\gamma$ value of $5 / 3$ (monatomic ideal gas), and $a_{0}=15 \mathrm{~km} \mathrm{~s}^{-1}$, the adiabatic sound speed for an ionized gas at $10^{4} \mathrm{~K}$. Most PNe have high densities and relatively slow shocks, so that the shocks are expected to be isothermal. Using $\gamma=1$ is therefore appropriate, and in Fig. 3 I show $\mathcal{R}$ as function of $u_{1}$ for this isothermal case, where I have used an isothermal sound speed of $11.7 \mathrm{~km} \mathrm{~s}^{-1}$, valid for an ionized gas of cosmic abundances at a temperature of $10^{4} \mathrm{~K}$. As expected from the limits derived above, the ratio is largest for low velocities. I should note that the ratio does depend on the choice for the sound speed $a_{0}$. A value corresponding to an electron temperature of $10^{4} \mathrm{~K}$ is typical for PNe, but the temperature can be both higher and lower than this, ranging from 5000 to $15000 \mathrm{~K}$. Lower temperatures give lower values of $\mathcal{R}$.

Looking at the data in Table 1, it is clear that for these $\mathrm{PNe}$, we are never in the very high Mach number regime. Using Fig. 3 one can see that for the observed ranges of velocities and for $\gamma=1$, the typical ratio $\mathcal{R}$ is between 1.3 and 1.5 , although it can be as high as 1.8 . Obviously this falls outside the formal errors of the method and becomes as important an effect as the geometric corrections applied for example by Li et al. (2002). Furthermore, the factor is always larger than 1, so it does not make sense to add a 20-30\% extra uncertainty to the distances. Rather, the distances should be scaled up by $30 \%$ and an extra error of $\sim 10 \%$ added to it. Figure 3 can be used to estimate



Fig. 2. The ratio $\mathcal{R}\left(=u_{\mathrm{s}} / u_{1}\right)$ as function of $u_{1}$ for the case $\gamma=5 / 3$ (no cooling), and (adiabatic) sound speed $a_{0}=15 \mathrm{~km} \mathrm{~s}^{-1}$. The six curves correspond to different values for the pre-shock velocity $u_{0}: 1$ (highest curve at $u_{1}=50 \mathrm{~km} \mathrm{~s}^{-1}$ ), 5, 10, 15, 20, and 25 (lowest curve) $\mathrm{km} \mathrm{s}^{-1}$.

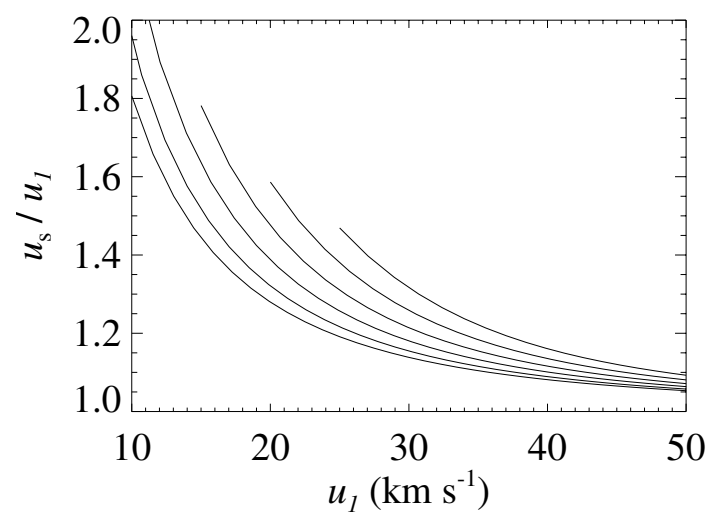

Fig. 3. The ratio $\mathcal{R}\left(=u_{\mathrm{s}} / u_{1}\right)$ as function of $u_{1}$ for the isothermal case $(\gamma=1)$, and (isothermal) sound speed $a_{0}=11.7 \mathrm{~km} \mathrm{~s}^{-1}$. The six curves correspond to different values for the pre-shock velocity $u_{0}$ : 1 (lowest curve), 5, 10, 15, 20, and 25 (highest curve) $\mathrm{km} \mathrm{s}^{-1}$.

the magnitude of this effect for individual PNe, which I will do in Sect. 5.

The conclusion then is that if the expansion parallaxes are measured from the shocked component of the PN, the distances should be multiplied by a factor $\mathcal{R}$ given by Eq. (4), where uncertainties in the velocity of the material into which the shock is expanding adds a $\sim 10 \%$ error. I will come back to this point in Sect. 5.

\section{Ionization fronts}

Ionization fronts are either R- or D-type. The basic difference is the speed of the front which for R-type fronts is higher than twice the sound speed in the neutral gas, giving the gas no time to react to the presence of the ionization front, wheras for D-type it is lower than twice the sound speed in the neutral gas. The result is that D-type fronts actually consist of a combination of an ionization front and a preceding shock front. A good overview of basic ionization front theory can be found in Shu (1992).

The numerical simulations for the formation of $\mathrm{PNe}$ which studied the effects of ionization fronts in detail, showed that although R-type fronts occur, they never persist for long, and it 


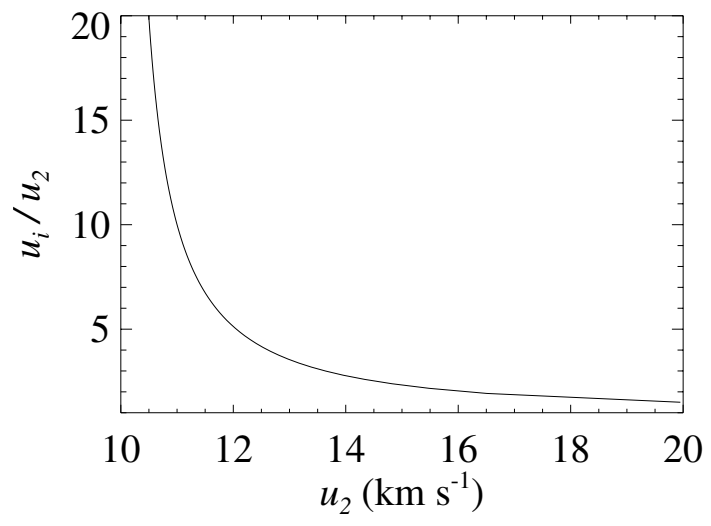

Fig. 4. The ratio $\mathcal{R}\left(=u_{\mathrm{i}} / u_{2}\right)$ as function of $u_{2}$ for a weak R-type ionization front running into a $10 \mathrm{~km} \mathrm{~s}^{-1}$ wind. The weaker the front the closer the ratio gets to one.

would be highly unlikely to observe one in action. When they occur, they are of the so-called weak type, i.e. supersonic with respect to both the neutral and ionized flow regions.

Weak D-type fronts (moving subsonically with respect to both the ionized and neutral regions) are more commonly found in the models, and persist for a longer time, and could be present in a number of observed PNe, although it is generally hard to prove this. The simulations also show that the presence of a D-type front sets up a disturbance in the gas which persists long after the front itself has disappeared, creating the shells which I discuss in the next section.

Basic ionization front theory shows that the velocities $v_{1}$ and $v_{2}$ on either side of the front are related by

$\frac{v_{1}}{v_{2}}=\frac{1}{2 a_{2}^{2}}\left(a_{1}^{2}+v_{1}^{2} \pm\left[\left(a_{1}^{2}+v_{1}^{2}\right)^{2}-4 a_{2}^{2} v_{1}^{2}\right]^{1 / 2}\right)$.

The indices 1 and 2 refer to the neutral and ionized sides, respectively. Just as in the previous section, $v$ refers to velocities in the frame of the discontinuity, and $u$ to velocities in the stellar frame. If the front moves with a velocity $u_{\mathrm{i}}$ in the stellar frame, the velocities on either side of it are $u_{1,2}=v_{1,2}+u_{\mathrm{i}}$. For the weak R-type fronts one has to use the - sign in Eq. (7) and for the weak D-type fronts the + . The velocity $v_{1}$ has to be larger than $v_{\mathrm{R}}=a_{2}+\left(a_{2}^{2}-a_{1}^{2}\right)^{1 / 2}$ for R-type fronts and smaller than $v_{\mathrm{D}}=a_{2}-\left(a_{2}^{2}-a_{1}^{2}\right)^{1 / 2}$ for D-type fronts.

In Fig. 4 I show the ratio $\mathcal{R}$, now defined as $u_{\mathrm{i}} / u_{2}$, against $u_{2}$ for weak R-fronts running into a medium with $u_{1}=10 \mathrm{~km} \mathrm{~s}^{-1}$. For the sound speeds I chose $a_{1}=1 \mathrm{~km} \mathrm{~s}^{-1}$ and $a_{2}=10 \mathrm{~km} \mathrm{~s}^{-1}$, and tests show that the ratio $\mathcal{R}$ is hardly sensitive to this choice. As can be expected this ratio is large for very fast moving fronts, and approaches 1 for the slower moving ones. Because of the large range of ratios, it would seem dangerous to apply any type of correction in case of R-type ionization fronts.

In Fig. $5 \mathrm{I}$ show the same ratio $\mathcal{R}$ against $u_{2}$ for weak $\mathrm{D}$-fronts, a situation which is more likely to occur in real PNe. Normally the shock front which precedes the D-front will have accelerated the AGB wind to a higher velocity. The numerical simulations show this velocity to be typically around $u_{1}=$ $20 \mathrm{~km} \mathrm{~s}^{-1}$. I plot the ratio for three values of $u_{1}$. Interestingly, around the typically observed velocities, the correction is of the same order of magnitude as for the shock waves in Sect. 2.

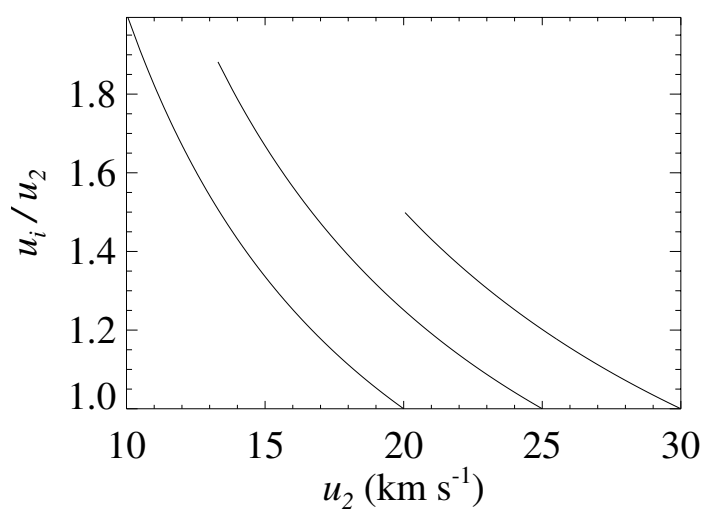

Fig. 5. The ratio $\mathcal{R}\left(=u_{\mathrm{i}} / u_{2}\right)$ as function of $u_{2}$ for a weak D-type ionization front running into a wind with velocity $u_{1}$ of (from left to right) 20,25 , and $30 \mathrm{~km} \mathrm{~s}^{-1}$.

The full solution for a D-type front would eliminate the choice for $u_{1}$. Shu (1992) showed how this can be done for a constant flux of ionizing photons impinging on a static and constant density environment. Attempts to do this for a stellar wind type environment have been only partly successful. Giuliani (1989) found a self-similar solution which requires the flux of ionizing photons to be time dependent and fall off as $t^{-1}$, which does not apply to most PNe.

Masson (1986, 1989a,b) applied a series of corrections to his determination of the distance to NGC 7027, BD+30 3639 and NGC 6591, which are partly related to the difference between the material velocity (which he referred to as the "bulk velocity"), and the (pattern) speed of the ionization front. The magnitudes of these corrections are actually similar to the ones found above, 1.1 to 1.2. Perhaps somewhat confusingly he applies these corrections to the measured angular expansion rate, so that the final figures he quotes for $\dot{\theta}$ are not actually the ones measured. He derives this correction from the fact that as the ionized shell expands, the density will go down, reducing the number of photons used up in recombinations, and allowing the ionization front to expand. This is a different approach to the one used above and does not use the jump conditions. However, the basic idea is still that the ionization front expands faster than the material flow of the gas, so the corrections are related. In not considering the jump conditions he implicitly assumes a weak R-type ionization front with a small density jump, and the correction corresponds therefore to the one from Fig. 4. The numerical simulations as well as the observed velocities show that this is not correct, although the correction factors come out at similar values. Note that Masson is the only author to actually apply any type of correction. In Sect. 5 I will comment some more on this.

The conclusion from this section is that it is more difficult to find a general solution for ionization fronts, which makes them less suitable than shock fronts for applying the expansion parallax method. However, the results in this section allow for corrections to be made, and below I will illustrate how these corrections could be applied. 




Fig. 6. The ratio $\mathcal{R}\left(=u_{\mathrm{s}} / u_{1}\right)$ as function of $u_{1}$ for the shell solutions from Chevalier (1997) and Shu et al. (2002). The solid line shows the solutions for a photo-ionized wind, the dotted line the same but with the influence of a wind included. The dashed line shows the result for photo-ionized winds with different density laws steeper than $r^{-2}$.

\section{Shells}

Numerical simulations show that the density disturbance induced by the D-type ionization front will persist and can be identified with the observed attached shells around the bright core nebula, such as for example in NGC 3242. Corradi et al. (2003) compiled a list of PNe with haloes and shells, which can serve as a reference. We follow their nomenclature and refer to these structures as shells.

Analytical models for the expansion of such shells are available in Chevalier (1997) and Shu et al. (2002), even though the latter had a different application in mind. Using their results, it is possible to extract a similar ratio $\mathcal{R}$ of pattern speed over material speed, where the pattern speed is now the movement of the edge of the shell. Since these models actually use the isothermal shock conditions, the results are basically identical to ones from Sect. 2. However, I list them separately, since shells are commonly observed and the Chevalier (1997) and Shu et al. (2002) results are the full solutions including the acceleration of the AGB wind after ionization.

For an AGB wind of constant mass loss and velocity, the results from Chevalier (1997) show that $\mathcal{R}$ is between 1.10 and 1.34. I plot this ratio against $u_{1}$ in Fig. 6, together with the ratio from his solution including the effect of a stellar wind, which gives somewhat lower values. The ratio for an AGB wind density falling off steeper $\left(\rho \propto r^{-\alpha}\right.$ with $\alpha>2$, Shu et al. 2002) are also shown.

The numerical simulations also show that when an attached shell forms, its velocity structure is that of a rarefaction wave, with a positive outward gradient. This means that the bright rim expanding into this will be moving into an area with a velocity lower than the original AGB wind velocity. The implication for the expansion parallax method is that when using the rims of PNe with attached shells a value of $u_{0}$ lower than $10 \mathrm{~km} \mathrm{~s}^{-1}$ is more appropriate when determining the correction factor from Sect. 2 .

The conclusion from this section is that for attached shells a correction of around $20 \%$ is needed.
Table 2. New distances.

\begin{tabular}{ll}
\hline \hline PN & Distance $(\mathrm{kpc})$ \\
\hline BD +303639 & $1.3 \pm 0.2$ \\
IC 2448 & $2.07 \pm 0.62$ \\
NGC 3242 & $0.55 \pm 0.23$ \\
NGC 6543 & $1.55 \pm 0.44$ \\
NGC 6578 & $2.90 \pm 0.78$ \\
NGC 6884 & $3.30 \pm 1.24$ \\
NGC 7027 & $0.68 \pm 0.17$ \\
NGC 7662 & $1.19 \pm 1.15$ \\
VY 2-2 & $4.68 \pm 1.20$ \\
\hline
\end{tabular}

\section{Implications for distances and the method}

The results presented thus far show that for the published distances, the results should typically be scaled up with a factor $1.3 \pm 0.2$, which interestingly enough eliminates the systematic discrepancy between the expansion parallax distances and statistical distances noted by Palen et al. (2002). In this section I will go through the list of PNe for which distances have been measured, consider corrections for each case, and give some suggestions on how to improve the usage of the expansion parallax method further. For calculating the corrections I use the values $\gamma=1$ and $a_{0}=11.7 \mathrm{~km} \mathrm{~s}^{-1}$ for the cases of shocks (corresponding to Fig. 3), $a_{1}=1$ and $a_{2}=10 \mathrm{~km} \mathrm{~s}^{-1}$ for ionization fronts (corresponding to Fig. 5). The first part of this section is intended not only to derive new distances, but also to illustrate how to derive and use the correction factors.

\subsection{New distance estimates}

Going through the list there are a number of PNe which contain bright rims and attached shells, and the rim can therefore be considered to be bounded by a shock. These are NGC 3242 , NGC 6578, NGC 6884, NGC 7662, and IC 2448. Making conservative assumptions about the value of $u_{0}\left(13 \pm 12 \mathrm{~km} \mathrm{~s}^{-1}\right)$ and using the results from Sect. 2, the distances to these PNe should be increased by factors $1.3 \pm 0.2,1.45 \pm 0.15,1.5 \pm$ $0.15,1.5 \pm 0.3$, and $1.5 \pm 0.1$ respectively. The uncertainties are due partly to the uncertainties in the reported spectroscopic velocities and partly to the uncertainty in the value of $u_{0}$. The latter could in principle be reduced if one assumes that the velocities in the shells follow the numerical models, in which case $u_{0}$ will be low $\left(<10 \mathrm{~km} \mathrm{~s}^{-1}\right)$. I list the new (conservative) values in Table 2.

NGC 6543 is a more complex nebula. The Chandra results show that it contains a wind-driven bubble, but the ionized material surrounding this bubble does not resemble a standard shell. This complicates the choice for $u_{0}$, but within reasonable limits the correction factor is $1.55 \pm 0.15$.

Three PNe in Table 1 are candidates for the presence of an ionization front: BD+30 3639, NGC 7027, and Vy2-2.

Of these BD+30 3639 is a single shell, and relatively young PN with molecular emission around it, although the deeper exposures also seem to show an ionized component around the 
bright shell (Sahai \& Trauger 1998). Kawamura \& Masson (1996) used a detailed analysis of the expansion of this object in order to derive the distance, assuming we are observing an ionization front. Part of this analysis is a correction factor of 1.19 to account for the difference between the material and pattern velocities, as mentioned in Sect. 3. Using the results from Sect. 3 for a weak D-type front, I estimate a correction factor of $1.2 \pm 0.2$ assuming a velocity of $25 \pm 5 \mathrm{~km} \mathrm{~s}^{-1}$ for the shocked neutral gas and a spectroscopic velocity of $22 \pm$ $4 \mathrm{~km} \mathrm{~s}^{-1}$ (as used by Kawamura \& Masson 1996), confirming their result for the distance.

Li et al. used optical HST data, better spectroscopic data and a correction for the ellipticity of the nebula to arrive at a smaller distance. Using their preferred value for the spectroscopic velocity, $25.6 \mathrm{~km} \mathrm{~s}^{-1}$, I find a correction factor of $1.1 \pm$ 0.1 if we are seeing an ionization front running into a shocked component with a velocity of $25.6-30 \mathrm{~km} \mathrm{~s}^{-1}$, and a correction of $1.3 \pm 0.1$ if we are seeing a shock. It therefore seems reasonable to increase the distance by $10 \%$ and to double the uncertainty on that number: $1.3 \pm 0.2 \mathrm{kpc}$, bringing it closer to the value from Kawamura \& Masson (1996)

NGC 7027 is a more complex PN where an ionization front has to be present, given the large amounts of dust and molecules surrounding this object. The elliptical shell seen at radio wavelengths (and partly obscured at optical wavelengths) could be the ionization front and is analyzed as such by Masson (1986, 1989a). To correct for the discrepancy between spectroscopic and angular velocities, he uses a factor of 1.2 in the first and 1.15 in the second paper (note that the second paper quotes $1 / 1.15=0.83$ as the correction factor). Further corrections for the decrease of the radio flux in the second paper actually largely cancel the effect of this factor.

Bains et al. (2003) analyzed high resolution optical long slit spectra of NGC 7027 and from the [O III] line derived an equatorial expansion velocity considerably lower than used by Masson (1989a), namely $13 \pm 1 \mathrm{~km} \mathrm{~s}^{-1}$. Cox et al. (2002) report K-band imaging and spectroscopy for NGC 7027. Their best fitting model has an equatorial expansion velocity in $\mathrm{Br} \gamma$ of about $13 \mathrm{~km} \mathrm{~s}^{-1}$ (Cox \& Huggins, private communication; this number is not given in the paper), consistent with the [O III] value. For the molecular emission $\left(\mathrm{H}_{2}\right)$ the same authors find an equatorial velocity of $\sim 15 \mathrm{~km} \mathrm{~s}^{-1}$. Allowing all possible values for the velocity of the neutral material $u_{1}$ (13-23 $\mathrm{km} \mathrm{s}^{-1}$ ), the correction factor would be $1.4 \pm 0.4$. Assuming that the $\mathrm{H}_{2}$ expansion is indicative of the value of $u_{1}$ $\left(13-17 \mathrm{~km} \mathrm{~s}^{-1}\right)$, the ratio becomes $1.2 \pm 0.2$. If the ionized shell is actually bounded by a shock, the results of Sect. 2 show that the correction factor would be $1.75 \pm 0.15$ for all allowed values of the pre-shock velocity $u_{0}$.

Taking an angular expansion of $4.84 \pm 0.82{\text { mas } \mathrm{yr}^{-1}}^{-1}$ (Masson 1989a, this includes the correction for flux variations $0.47 \pm 0.47$ ), but not the correction factor of 0.83 ), combining this with an equatorial velocity of $13 \mathrm{~km} \mathrm{~s}^{-1}$, and applying a correction of $1.2 \pm 0.2$, I arrive at a new distance of $680 \pm$ $170 \mathrm{pc}$. Note that this error is rather optimistic in view of the wide range of correction factors mentioned above.

Vy2-2 is a compact PN which is hard to categorize. Also here one may have to be aware of ionization fronts since this is a fairly young, low excitation PN. Assuming either an ionization front or an isothermal shock, the reported distance should be scaled up by $1.3 \pm 0.3$

Two PNe in the list are hard to categorize in the standard wind-blown bubble plus photo-ionization scheme: NGC 6210 and NGC 6572. I therefore will not suggest any corrections for these, although shocks could very well be present. For NGC 6210 this would imply a $30 \%$ increase in the reported distance, but for NGC 6572 the correction could become very large (1.4-1.8) due to the low value of the reported spectroscopic velocity.

\subsection{Improving the method}

The correction factors derived in this paper depend on a number of parameters. As already indicated, due to the isothermal nature of the slow shocks in PNe, the preferred value for the adiabatic index $\gamma$ is 1 . The sound speed in the ionized medium $\left(a_{0}\right)$ I have taken to be $11.7 \mathrm{~km} \mathrm{~s}^{-1}$ in Fig. 3, which is the value for an electron temperature of $10^{4} \mathrm{~K}$. If the PN is known to have a particularly high or low electron temperature the figure should be recalculated for the appropriate sound speed, since the values for $\mathcal{R}$ unfortunately do depend on the choice of the sound speed. The appropriate sound speed to use here is the isothermal sound speed.

Figure 3 or its equivalent can then be used to find the correction factor for the range of values of $u_{0}$ and $u_{1}$ which seem reasonable. Observational data on $u_{0}$ is scarce, but if it cannot be further constrained, $10 \pm 10 \mathrm{~km} \mathrm{~s}^{-1}$ should cover most cases. For $u_{1}$ the question is which spectroscopic velocity to pick. Ultimately, for an individual PN this question can only be answered through detailed (photo-ionization or hydrodynamic) modelling of the PN, assuming it is actually possible to produce a unique model for it. The approach of Gesicki et al. (1998), who derive spatial velocity profiles using photoionization models, may be useful here. Generic hydrodynamic modelling may help establish what would be a good choice in general cases and I understand that a project to do this is under way (Schönberner, private communication). Until that time the choice for $u_{1}$ will introduce uncertainties in the method, as it has always done. It is important to realize that the jump conditions are valid just before and after the front, so the best choice would be a velocity as close as possible to the front.

Round or elliptical PNe with attached shells are particularly suitable for the expansion parallax method. Their inner bright rim is bounded by a shock, and the presence of a surrounding ionized shell shows that this is not an ionization front. For these almost round $\mathrm{PNe}$, long slit spectroscopy can give a good indication of the spatial and velocity structure, since it will be quite similar in the directions perpendicular and parallel to the line of sight. This way a distinction can be made between the velocity of the rim and the shell (the latter can be both higher and lower than that of the rim). If possible it is best to choose species which lie closest to the outer edge of the rim so as to avoid any velocity variations within the rim. For these rims numerical models show that they are expanding into a region of low velocity, typically lower than that of the 
original AGB wind. Choosing a value for $u_{0}$ of around $5 \mathrm{~km} \mathrm{~s}^{-1}$ is a reasonable guess. In any case, the corrections as derived in Sect. 2 only weakly depend on this choice. Using the measured spectroscopic velocity, the ratio $\mathcal{R}$ can be determined.

It would be interesting to derive an independent distance using the attached shells, if available. If the long slit spectroscopy provides a velocity for the shell, then the corrections from Sect. 4 can be used to find a distance. Models show that the velocities of the shells are not as strongly position dependent as those of the inner rims, something which is reflected in the only mild ellipticities found observationally. This may make the outer edges of shells actually better suited for distance determinations with the expansion parallax method.

It is best to avoid areas and features well away from where a shock is suspected. At least for a shock we can make a connection between the pattern and material velocities. Away from these discontinuities, the two will also differ, but there is no way to know how.

Bipolar PNe display a larger range of expansion velocities, which means that it is harder to correct for inclination effects. Palen et al. (2002) state that the method is unusable for extreme bipolars, which seems too pessimistic. However, a thorough understanding of the dynamics of the complete PN is essential to be able to apply the method to these PNe, and in most cases this information is not available. It would also be valuable to have more radiation-hydrodynamic modelling of such systems.

\section{Conclusions}

Pattern velocities and the material (or bulk) velocities in a gas are not necessarily the same. For discontinuities, such as shocks and ionization fronts, the relation between the two can easily be derived using the jump conditions across them. The pattern velocity is then found to be always higher than the material velocity. Since measuring the expansion of PNe in the sky is mostly done using sharp edges, which are associated with either shocks or ionization fronts, a correction should be applied before calculating the distance from the ratio of the two velocities. This correction is typically larger for velocities of the order one or two times the sound speed in the ionized material, which is actually what is measured in most PNe.

Not using this correction will systematically underestimate the distances to PNe. For the sample of PNe to which the expansion parallax method has been applied successfully, the correction factors are around 1.2 to 1.3 for both shocks and ionization fronts. Applying the corrections given in this paper should lead to improved distance determinations to PNe.

Acknowledgements. I like to thank Pierre Cox and Patrick Huggins for providing me with a value for the equatorial expansion velocity of NGC 7027. My research has been made possible by a fellowship of the Royal Netherlands Academy of Arts and Sciences.

\section{References}

Bains, I., Bryce, M., Mellema, G., Redman, M. P., \& Thomasson, P. 2003, MNRAS, 340, 381

Chevalier, R. A. 1997, ApJ, 488, 263

Christianto, H., \& Seaquist, E. R. 1998, AJ, 115, 2466

Corradi, R. L. M., Schönberner, D., Steffen, M., \& Perinotto, M. 2000, A\&A, 354, 1071

Corradi, R. L. M., Schönberner, D., Steffen, M., \& Perinotto, M. 2003, MNRAS, 340, 417

Cox, P., Huggins, P. J., Maillard, J.-P., et al. 2002, A\&A, 384, 603

Gesicki, K., Zijlstra, A. A., Acker, A., \& Szczerba, R. 1998, A\&A, 329,265

Hajian, A. R., \& Terzian, Y. 1996, PASP, 108, 419

Hajian, A. R., Terzian, Y., \& Bignell, C. 1993, AJ, 106, 1965

Hajian, A. R., Terzian, Y., \& Bignell, C. 1995, AJ, 109, 2600

Kawamura, J., \& Masson, C. 1996, ApJ, 461, 282

Li, J., Harrington, J. P., \& Borkowski, K. J. 2002, AJ, 123, 2676

Marten, H., Gesicki, K., \& Szczerba, R. 1993, in Planetary Nebulae, IAU Symp. 155, 315

Marten, H., \& Schoenberner, D. 1991, A\&A, 248, 590

Masson, C. R. 1986, ApJ, 302, L27

Masson, C. R. 1989a, ApJ, 336, 294

Masson, C. R. 1989b, ApJ, 346, 243

Mellema, G. 1994, A\&A, 290, 915

Mellema, G. 1995, MNRAS, 277, 173

Palen, S., Balick, B., Hajian, A. R., et al. 2002, AJ, 123, 2666

Reed, D. S., Balick, B., Hajian, A. R., et al. 1999, AJ, 118, 2430

Sahai, R., \& Trauger, J. T. 1998, AJ, 116, 1357

Schönberner, D., \& Steffen, M. 2003, in Planetary Nebulae, Their Evolution and Role in the Universe, IAU Symp., 209, in press

Shu, F. H. 1992, Physics of Astrophysics, Vol. II (University Science Books, ISBN 0-935702-65-2)

Shu, F. H., Lizano, S., Galli, D., Cantó, J., \& Laughlin, G. 2002, ApJ, 580, 969

Steffen, M., Schonberner, D., Kifonidis, K., \& Stahlberg, J. 1997, in Planetary Nebulae, IAU Symp., 180, 368

Terzian, Y. 1993, in Planetary Nebulae, IAU Symp., 155, 109 